\title{
Genetic testing of adults with intellectual disability
}

\author{
Jana de Villiers, ${ }^{1}$ Mary Porteous ${ }^{2}$
}

The Psychiatrist (2012), 36, 409-413, doi: 10.1192/pb.bp.111.038216

1Lynebank Hospital, Dunfermline; ${ }^{2}$ Western General Hospital, Edinburgh Correspondence to Jana de Villiers (janadevilliers@nhs.net)

First received 15 Dec 2011, final revision 25 May 2012, accepted 13 Jun 2012
Method Patients known to learning disability services in two health boards in southeast Scotland were cross-matched with the patients tested at the Western General Hospital in Edinburgh. Those with a positive genetic diagnosis were identified. Semistructured interviews were conducted with senior learning disability psychiatrists and clinical genetics consultants.

Results Of the 3323 patients with intellectual disability across both health boards, $41 \%$ have had genetic tests and $6 \%$ have an identified genetic abnormality as the cause for their intellectual disability. Of the 1349 patients who have been tested, a genetic abnormality was found in $14 \%$. Psychiatrists named several benefits to genetic testing, but they also highlighted a number of non-medical reasons for not testing adults with intellectual disability.

Clinical implications Identifying genetic aetiology in intellectual disability has a number of benefits. Our study would indicate that genetic diagnoses are being missed due to a lack of genetic testing in this patient group. Adult learning disability services need to consider increasing genetic testing.

Declaration of interest None.
Intellectual disability is a common condition, affecting $1.5-4 \%$ of the population (with higher rates in low- and middle-income countries). It consists of a heterogeneous group of clinical conditions with different aetiologies, including genetic (e.g. fragile-X syndrome), nutritional (e.g. iodine deficiency), infectious (e.g. intrauterine rubella), metabolic (e.g. phenylketonuria) and neurotoxic conditions (e.g. fetal alcohol syndrome). ${ }^{1}$ There have been notable technical advances in the identification of genetic abnormalities in the past few years. In 1959, the first evidence linking genomic imbalances to human disease was gathered when Down syndrome was shown to be caused by the presence of an extra chromosome 21 . Chromosome banding techniques were developed in the 1970s, initially with a resolution of 3-5 MB. Fluorescent in situ hybridisation (FISH) enabled even smaller alterations to be identified (resolution 50-100 KB). The next major development was array comparative genomic hybridisation (array CGH). This technology has the capacity to examine the whole human genome on a single chip to a resolution of a few hundred base pairs.

As the technology has improved, diagnostic yields in patients with intellectual disability have increased. With conventional cytogenetics, $3-4 \%$ of abnormalities were identified, increasing to $5-7 \%$ with subtelomere FISH. Micro-array is able to identify clinically relevant copy number variations (CNVs) in $15-20 \%$ of patients with unexplained intellectual disability. ${ }^{2}$ A recent study ${ }^{3}$ of 45 patients with unexplained intellectual disability (18 men and 27 women, with an average IQ of 60 and an average age of 35 years) found pathogenic array CGH findings in $22 \%$. Of note, those with positive findings tended to have multiple mild dysmorphic features, which were generally not noted prior to testing. The authors reported a 'general sense of satisfaction from family members that a biological explanation was now evident as the cause of their relative's intellectual disability'.

Research on the perceptions of adult patients with intellectual disability (and their families) of genetic testing highlights the positive view of genetics held, in contrast to some intellectual disability literature and the views expressed by some professionals who suggest that genetic advances may have negative consequences for individuals with intellectual disability. ${ }^{4}$ Identifying a genetic aetiology for intellectual disability has a number of practical advantages. These include improving patient management by addressing known comorbidities. For instance, up to $65 \%$ of patients with Cornelia de Lange syndrome have pathological gastro-oesophageal disease (GORD) and there is a correlation between behavioural symptoms and oesophageal damage. Behavioural symptoms improve once the GORD is treated. ${ }^{5}$ Surveillance for serious comorbidities, including giant cell astrocytomas in people with tuberous sclerosis, becomes possible.

A genetic diagnosis allows the patient and family to access support networks and further information, including prognostic information. Increased resources become available, such as managed care networks or support teams for specific conditions. 
Little is known about the use of genetic testing in routine practice by learning disability services in the UK.

Recent studies indicate that in approximately $20 \%$ of patients intellectual disability has a genetic aetiology. This study was undertaken to identify the proportion of adults with intellectual disability known to learning disability services in south-east Scotland who have had genetic tests, and to determine the percentage of patients with a genetic diagnosis known to services. Owing to a perceived low uptake of genetic testing, senior learning disability psychiatrists and clinical genetics consultants were interviewed to clarify the reasons for an apparent reluctance to investigate for genetic abnormalities in adults with intellectual disability.

\section{Method}

Each health board area in the south-east of Scotland provides a multidisciplinary learning disability service, and clinical contact by any member of the team is logged in a database (Patient Information Management System). The total population covered by the two health boards in this study (NHS Lothian and NHS Borders) was approximately 900000 people in 2009. The region is a mix of urban and rural areas. The study sample consisted of any patient that was seen by a member of the learning disability service in 2009, so as to identify active cases. Patient details were cross-matched with the databases of patients who have had genetic tests done in any of the genetic laboratories located at the Western General Hospital in Edinburgh since 1994. All genetic testing for patients in south-east Scotland is undertaken at the Western General Hospital. Duplicate entries (for those patients tested on more than one occasion or in different laboratories) were identified. The proportion of patients known to learning disability services who have been tested was calculated, and those with a positive genetic diagnosis were identified. The proportion of patients known to adult learning disability services who have a known genetic aetiology for their intellectual disability was then calculated.

Semi-structured interviews were conducted with 8 (out of 12) senior learning disability psychiatrists (2 associate specialists and 6 consultants; 12 senior consultants are employed across both health boards, some part-time) and 2 (out of 4) clinical genetics consultants. Those interviewed were senior clinicians who agreed to participate in the project. They were aware that the project was related to the genetic testing of adults with intellectual disability, but had no prior knowledge of the questions that would be asked. All the learning disability psychiatrists worked in adult-only services. The interviews were digitally recorded and transcribed by one of the authors (J. de V.). The questions asked are listed in Box 1 . The responses were collated by J. de V., with key points and common views identified.

The South East Scotland Research Ethics Service has confirmed that this research project did not need National Health Service (NHS) ethical review, as it only used data obtained as part of usual care and was an opinion survey of NHS staff on service delivery and development.
Box 1 Questions for clinicians in the study

- Do you consider genetic testing to be a routine part of assessment in someone with intellectual disability?

- How many patients have you tested for genetic abnormalities in the past year?

- What clinical features might prompt you to consider genetic testing?

- How useful do you consider genetic testing?

- Do you have any objections to genetic testing?

- What benefits/disadvantages are associated with genetic testing?

- Are there ethical issues to consider?

- Any other comments?

\section{Results}

In 2009, 3323 patients were receiving learning disability services across both health boards. Of these, 1349 (41\%) have had genetic testing at some point since 1994 and 189 (6\%) had an identified genetic abnormality as the cause for their intellectual disability; 189 (14\%) of the 1349 patients who have had genetic testing had a genetic abnormality identified.

A number of areas of concern emerged from the interviews. Five of the eight learning disability psychiatrists did not think that genetic testing should be a routine part of the assessment of adults with intellectual disability, and some respondents commented that they have never requested such testing. Both clinical genetics consultants felt that it should be routine. Of those doctors who did not think that testing should be routine, only one had requested genetic testing in a patient in the year prior to the interview. By contrast, the three psychiatrists who did consider testing to be part of routine assessment had requested between three and six genetic tests each the previous year.

Four of the eight psychiatrists had objections to genetic testing. The most cited concerns were about the patient's capacity to consent to testing and the ethics of obtaining blood samples from those either unable to consent or who experience distress during venepuncture. Where patients do not have capacity, the lack of clarity about the results having direct benefit to the patient rather than their family was felt to be problematic, particularly if a test result had implications for the wider family. A couple of psychiatrists cited cases where the patient had either no contact or difficult relationships with the wider family, and the complexities that could arise if a genetic test result required discussions with the family. One psychiatrist highlighted the possible non-medical use of the information, for example by insurance companies (although the misuse of information in this way is illegal in the UK). There were also concerns that it may lead to discrimination and therapeutic fatalism. Some expressed the view that genetic testing did not have an impact on day-to-day management and questioned its utility. The possibility of society valuing people with genetic disorders less and that testing could lead to parents choosing 'designer babies' was also raised. Some learning 
disability psychiatrists commented that they felt they lacked adequate knowledge about genetics and how best to approach the issue of testing.

Some benefits to testing were described, including: clarity of diagnosis, access to information regarding the abnormality, the ability to build up knowledge of symptoms and signs, increased clinical vigilance for known associations, to help clarify prognosis, and to assist families in their planning.

Practical barriers to testing that were mentioned included family or patient resistance, difficulty in obtaining blood tubes and request forms, lack of referral for or consideration of genetic testing, obtaining consent and avoiding patient distress during sample collection. A selection of doctors' comments is given in Box 2 (geneticists) and Box 3 (psychiatrists).

\section{Discussion}

The findings from this study indicate that $6 \%$ of patients known to adult learning disability services in the study area have a known genetic abnormality. Most of the patients in the sample who have had genetic testing were tested as children, or before the recent availability of array CGH (currently considered the investigation of choice in those with intellectual disability). Studies ${ }^{2,3,6,7}$ and experience at the Clinical Genetics Department at the Western General Hospital in Edinburgh indicate that approximately $20 \%$ of patients with intellectual disability of unknown cause have pathogenic genetic abnormalities. It is likely that approximately $14 \%$ of the study population who have intellectual

\section{Box 2 Clinical geneticists' comments}

\section{Clinical geneticist consultant 1 :}

'I think it can be useful for the adults themselves sometimes to actually get a name for the problems that they've had.

'I think it's important always to ask why the tests are being done and to make sure that it's of particular clinical benefit to the patient or to the patient's family because I think that if the patient has a relationship with their family then the effect on the family is broadly similar to the effect on the patient. They should be considered as a unit, not as two separate stakeholders.'

'We need to develop better protocols so that we can offer testing without it being a big drama. The testing is very seldom urgent so it's important to take time and get it right.'

Clinical geneticist consultant 2:

'Almost anyone with moderate to severe learning disability should be investigated'.

'I think it is easy to underestimate how important it is to people themselves to have a diagnosis.'

'For children there is a very good service but adults perhaps don't get quite the same level of genetic and dysmorphology input that they should do.'

\section{Box 3 Learning disability psychiatrists' comments}

Learning disability psychiatrist 1 :

I think there is a great disparity in the genetic service that is being offered by us as psychiatrists in learning disability, between children and adults.'

Learning disability psychiatrist 4:

'The disadvantages of genetic testing are that you might find things you'd ... rather wish you didn't know.'

Learning disability psychiatrist 5:

'I think I would probably welcome clearer guidance and criteria. [I feel I have a] lack of knowledge as to what sort of tests I would need to do and how I would actually go about it.'

'. . . it might be satisfying for doctors and clinicians to know what's what, but really is it of any benefit to that individual or their family?'

disability due to genetic abnormalities have not been identified by services. There is a lack of data on the uptake of genetic testing in this patient group, but informal contact with colleagues would indicate that clinical practice is not markedly different in other services within the UK.

The interviews revealed that the low uptake of testing by senior learning disability psychiatrists appears to be largely for non-medical reasons. Of particular note is that some senior psychiatrists have never requested genetic testing despite working with a patient population of whom approximately $20 \%$ will have a genetic abnormality.

The lack of capacity to consent to genetic testing was the most commonly expressed barrier to genetic testing. Some patients will be able to consent in their own right, but many will lack the capacity to understand the investigations. Legal provisions are available to sanction investigations in these circumstances. However, some patients with intellectual disability, and in particular those with autism, can experience considerable distress during phlebotomy. Professionals are therefore understandably reluctant to undertake investigations which may not have clear immediate clinical benefits to patients. Additionally, doctors within the learning disability field are cautious of undertaking investigations on behalf of the wider family, as these may seem exploitative and family dynamics are often complex. For patients who experience extreme distress during phlebotomy but who require investigations, current practice includes obtaining samples when the patient receives sedation or anaesthesia for an unrelated medical or dental procedure. Consideration could be given to obtaining blood samples for genetic testing in this manner should an opportunity arise.

Many professionals working with patients with intellectual disability adhere to the social model of disability (which focuses on maximising the potential of the individual through education, social work and psychology) and have little interest in the aetiology of the intellectual disability. Diagnosing underlying genetic abnormalities is often viewed as being irrelevant to the management of the patient, or is seen as being potentially negative (although, as 
Ward et al have commented, ${ }^{8}$ the voices of those most affected by developments in genetics, namely people with intellectual disability and their families, have been 'conspicuously absent'). A recent study of research participant and family perspectives on genetic diagnoses ${ }^{4}$ interviewed people who had agreed to participate in the Genetics of Learning Disability (GOLD) study undertaken by the University of Cambridge and the Wellcome Trust. The majority (56\%) cited obtaining an explanation for the family's difficulties as the reason for participating, with $47 \%$ wanting to enable other families to have choices about reproduction. Respondents (who were mainly families of people with intellectual disability, rather than adults with intellectual disability themselves) repeatedly stressed the importance of obtaining a definitive diagnosis.

A study of caregiver and adult patient perspectives on the importance of a diagnosis of 22q11.2 deletion syndrome obtained similar results. ${ }^{9}$ Both patients and caregivers felt that the diagnosis, even if occurring later in life, was a critical event with many positive consequences. The cited benefits included greater understanding and certainty, a sense of purpose, and increased opportunities to optimise the care and support that they receive. Currently, 22q11.2 deletion syndrome remains underdiagnosed, even though its identification can lead to significant changes in medical management, follow-up and genetic counselling. The authors note that testing uptake may be particularly low for adults and/or for those with milder forms of intellectual disability. Many respondents reported feeling frustrated and powerless due to the years of uncertainty about the diagnosis, and felt that the lack of an accurate diagnosis led to mismanagement (owing to medical conditions being dealt with in isolation or behavioural problems being misattributed). The known association between 22q11.2 deletion syndrome and treatable psychiatric illnesses (such as schizophrenia and anxiety disorders) is particularly important in those with intellectual disability, in whom clinical diagnosis of mental illness may be more challenging. Patients and relatives may regard genetic diagnoses differently from professionals, and the diagnostic certainty in and of itself appears closely linked to psychological benefit for the patient and the family. This particular study demonstrated that the benefits of diagnosis extend to the adult with intellectual disability, and not just to family members.

The uneasiness concerning syndrome diagnosis expressed by professionals working in the field of intellectual disability may stem from the belief that a genetic diagnosis merely adds a 'label' and could potentially have harmful consequences for people with intellectual disability (the negative effects of the eugenics movement in the early 20th century being a powerful reminder of the abuse and misuse of scientific advances). ${ }^{10}$ However, in the study by Costain et $a l,{ }^{9} 61 \%$ of the original cohort of patients and caregivers referred to a specialist service responded to a further enquiry, reporting no deleterious effects as a result of a genetic diagnosis.

The benefits of identifying a genetic disorder extend to people with fragile- $\mathrm{X}$ syndrome and their families. Fragile- $\mathrm{X}$ syndrome lacks distinctive defining features at birth and it is not unusual for diagnoses to be delayed, often into adulthood. It demonstrates the need for increased testing, as an unknown number of cases remain undetected and there are important implications for the wider family. Testing in adulthood would inform relatives of their possible carrier status and allow the patient and family to access information. There are health implications for relatives if they are premutation carriers (55-200 repeats), with females at risk of premature ovarian failure, and both males and females at risk of developing ataxia later in life.

In a 2003 study by Skinner et al, ${ }^{11}$ which looked at parental attitudes to screening for fragile-X syndrome, parents strongly favoured carrier screening for women, and newborn screening for both affected and carrier status, on a voluntary basis. Their views are at odds with the more conservative approaches of human genetics and public health professionals, who do not advocate testing for carrier status in children, or for genetic disorders with no medical cure or proven intervention. The families involved in the study experienced first-hand the psychological and social ramifications of an inherited genetic disorder, and their views add to the debate about who has the right to know what and when. ${ }^{11}$

The Joint Committee on Medical Genetics noted in 2005 that: 'Clinical experience confirms that the majority of people are willing to undergo investigations to help other family members, even if there is no direct medical benefit to themselves. The Genetic Interest Group has argued strongly that it should not be presumed that an adult with incapacity would be less altruistic than a competent adult in wishing to assist other family members in genetic investigations. ${ }^{2}$

The reluctance of professionals to undertake genetic testing can appear paternalistic, with clinicians wanting to protect patients and their families from potentially difficult issues. However, a survey of lay comments analysed by Bailey et $a l^{13}$ demonstrated that non-professionals viewed genetic screening as beneficial to children and families. Aside from the direct benefits to the child affected, benefits to the family cited included 'sparing them the pain, anxiety, and emotional toll that comes from not knowing what is wrong with their child, and saving them from the diagnostic odyssey'. In contrast to professional views, lay commentators took the firm stance that 'there is substantial value in knowing', even for medically untreatable conditions. The authors conclude that professionals tend to endorse a more conservative approach to screening, whereas family members and advocacy groups take a broader view of the potential benefits. These debates are relevant to adults with intellectual disability, since some professionals equally view the genetic testing of someone with an 'untreatable' condition as futile. The person's family may well have a different view.

Concerns related to non-medical use of information (which is illegal in the UK), discrimination and therapeutic fatalism seem misplaced. Clinicians are commonly involved in the care of patients with Down syndrome, Prader-Willi syndrome and fragile- $\mathrm{X}$ syndrome without these concerns being borne out.

Are there any clinical symptoms or signs that can guide genetic testing? It is generally recognised that an unusual appearance and a high number of anomalies (more than six) indicate a possible chromosome abnormality, yet four in ten 
patients with intellectual disability attributable to chromosome abnormalities have no dysmorphic features. On this basis, routine cytogenetic testing is recommended in children with developmental delay even if no dysmorphic features or clinical features suggestive of a syndrome are present. $^{14,15}$ Therefore, it could be argued that all adults with intellectual disability of unknown origin should be considered for genetic testing, in particular for array $\mathrm{CGH}$, even if no specific physical features are present.

Given the advances in genetic testing, and increasing knowledge of the role of genetics in relation to physical and mental health, it is our view that adult learning disability services need to consider genetic testing in all patients with intellectual disability of unknown aetiology. As a result of this project, an assessment pathway has been agreed locally that any adult with intellectual disability referred for genetic testing would have array CGH plus genetic probes of common syndromes (referred to locally as an intellectual disability screen) applied to their samples. If an abnormality is identified, the Clinical Genetics Department meets with the patient and family to discuss the implications. For specific syndromes, information on support groups and specialist treatment services is provided.

There are clear benefits to identifying those with a genetic cause for their intellectual disability, as outlined earlier. Recent trials of potential treatments for specific genetic disorders (such as mGluR5 antagonists for fragile- $\mathrm{X}^{16}$ ) hold the exciting promise that the benefits of diagnosis could soon include targeted therapy and improved outcomes.

\section{Acknowledgements}

We would like to thank Professor David Fitzpatrick, Dr Andrew McKechanie, Dr Alan Carson and Dr Andrew Stanfield for their help with this project.

\section{About the authors}

Jana de Villiers is a consultant psychiatrist for the Fife Forensic Learning Disability Service, Lynebank Hospital, Dunfermline, UK. Mary Porteous is a consultant clinical geneticist and Service Lead for the South East Scotland Genetic Service, Department of Clinical Genetics, Western General Hospital, Edinburgh, UK.

\section{References}

1 Salvador-Carulla L, Bertelli M. 'Mental retardation' or 'intellectual disability': time for a conceptual change. Psychopathology 2008; 41: 10-6.

2 Li MM, Andersson HC. Clinical application of microarray-based molecular cytogenetics: an emerging new era of genomic medicine. J Pediatrics 2009; 105: 311-7.

3 Taylor MRG, Jirikowic J, Wells C, Springer M, McGavran L, Lunt B, et al. High prevalence of array comparative genomic hybridization abnormalities in adults with unexplained intellectual disability. Genet Med 2010; 12: 32-8

4 Statham H, Ponder M, Richards M, Hallowell N, Raymond FL. A family perspective of the value of a diagnosis for intellectual disability: experiences from a genetic research study. Br J Learn Disabil 2011; 39: 46-56.

5 Berg K, Arron K, Burbridge C, Moss J, Oliver C. Carer-reported contemporary health problems in people with severe and profound intellectual disability and genetic syndromes. J Policy Practice Intellect Disabil 2007; 4: 120-8.

6 Ropers HH. Genetics of intellectual disability. Curr Opin Genet Develop 2008; 18: 241-50

7 Stankiewicz P, Beaudet AL. Use of array CGH in the evaluation of dysmorphology, malformations, developmental delay and idiopathic mental retardation. Curr Opin Genet Develop 2007; 17: 182-92.

8 Ward L, Howarth J, Rodgers J. Difference and choice: exploring prenatal testing and the use of genetic information with people with learning difficulties. Br J Learn Disabil 2002; 30: 50-5.

9 Costain G, Chow EWC, Ray PN, Bassett AS. Caregiver and adult patient perspectives on the importance of a diagnosis of 22q11.2 deletion syndrome. J Intellect Disabil Res 2012; 56: 641-51.

10 Holland A, Clare I. The human genome project: considerations for people with intellectual disabilities. J Intellect Disabil Res 2003; 47: 515-25.

11 Skinner D, Sparkman KL, Bailey DB. Screening for Fragile X Syndrome: parent attitudes and perspectives. Genet Med 2003; 5: 378-84.

12 Royal College of Physicians, Royal College of Pathologists, British Society for Human Genetics. Consent and Confidentiality in Genetic Practice: Guidance on Genetic Testing and Sharing Genetic Information. Report of the Joint Committee on Medical Genetics. RCP, RCPath, BSHG, 2005.

13 Bailey DB, Beskow LM, Davis AM, Skinner D. Changing perspectives on the benefits of newborn screening. Ment Retard Dev Disabil Res Rev 2006; 12: 270-9.

14 Graham SM, Selikowitz M. Chromosome testing in children with developmental delay in whom the aetiology is not evident clinically. J Paediatr Child Health 1993; 29: 360-2.

15 Moeschler JB, Shevell M. Clinical genetic evaluation of the child with mental retardation or developmental delays. Pediatrics 2006; 117 2304-16.

16 Jacquemont $S$, Curie $A$, des Portes $V$ Torrioli MG, Berry-Kravis $E$ Hagerman RJ, et al. Epigenetic modification of the FMR1 gene in Fragile $X$ syndrome is associated with differential response to the mGluR5 antagonist AFQ056. Sci Transl Med 2011; 3: 64ra1. 